\title{
Light-Weight Routing Protocol in IoT-Based Inter-Device Telecommunication Wireless Environment
}

\author{
Hayoung $\mathrm{Oh}^{1}$, Sangsoon $\mathrm{Lim}^{2}$ \\ ${ }^{1}$ School of Electronic and Engineering, SoongSil University, Seoul, South Korea \\ ${ }^{2}$ Software R\&D Center, Samsung Electronics, Seoul, South Korea
}

\begin{tabular}{l} 
Article Info \\
\hline Article history: \\
Received Mar 15, 2016 \\
Revised Jun 1, 2016 \\
Accepted Jun 15, 2016 \\
\hline
\end{tabular}

Keyword:

Hyper-connectivity IoT (internet of thing) Light-weight routing protocol RPL

\begin{abstract}
The primary task for IoT-based hyper-connectivity communications lies in the development of direct communications technique among IoT devices in RPL (Routing Protocol for Low-Power and Lossy Networks) environment without the aid from infras such as access points, base stations etc. In a lowpower and lossy wireless network, IoT devices and routers cannot keep the original path toward the destination since they have the limited memory, except for a limited number of the default router information.. Different from the previous light-weight routing protocols focusing on the reduction of the control messages, the proposed scheme provides the light-weight IPv6 address auto-configuration, IPv6 neighbor discovery and routing protocol in a IoT capable infra-less wireless networks with the bloom filer and enhanced rank concepts. And for the first time we evaluate our proposed scheme based on the modeling of various probability distributions in the IoT environments with the lossy wireless link. Specifically, the proposed enhanced RPL based light-weight routing protocol improves the robustness with the multi-paths locally established based on the enhanced rank concepts even though lossy wireless links are existed. We showed the improvements of the proposed scheme up to $40 \%$ than the RPL based protocol.
\end{abstract}

Copyright (C) 2016 Institute of Advanced Engineering and Science. All rights reserved.

\section{Corresponding Author:}

Sangsoon Lim, Software R\&D Center, Samsung Electronics, 56, Seongchon-gil, Seocho-gu, Seoul, Korea. Email: lssgood80@gmail.com

\section{INTRODUCTION}

The rising demand for various internet services and the recent development in the field of smart electronic devices has been facilitating the movement of internet environment from the human-centered settings to IoT (Internet of Things) settings full of intelligent devices. In this trend, IoT-applicable terminals are required to be low-power and light-weight and thus sensor networks are the firsthand candidate for IoT networking (while the sensor network has been so far a sort of closed network for individual utilization). If sensor-based information is connected to smart devices such as smart phones, smart TVs etc., a new service focusing on individual life amenities will be available. Since it is projected that 24 billion terminals will be connected to the internet by 2020, the IoT-based services will be expanded to vast scope of areas including smart-home, smart grid, intelligent health care, intelligent transportation service etc [1]-[4].

In this regard, the sensor capability of an individual product has been remarkably expanding as much as the recent smart devices are equipped with accelerometers, GPS sensors, proximity sensors, camera etc. These new sensor devices provide internal sensory values that may be utilized for new application services and new framework accessibilities. However, these built-in sensors are limited in size, price and space availability and hence may not be put into one device. To meet the ever-increasing demand for lifeconvenience smart services, the inter-connection of IoT networks is emerging as the most suitable solution in 
that this approach enables a smart device to make use of external sensors for information collection and remote control. Hence, the relevant industries are engaged in hot pursuit for technology development and research activities. For instance, smart phones are constantly roving along with the movement of the users; they may collect information from the stationary sensors around proximate areas and return the useful environmental information such as real time temperature and humidity etc.

The three major IoT technologies are sensing technology, wire-and wireless communications \& network infra technology and service interface technology. The sensing technology collects, processes and manages information from sensors and support transformation of such information for pre-determined services. To enhance IoT services associated with end-to-end networks, the developer needs various communications and networking technologies including proximity communication technology (WPAN, WLAN etc) [5],[6], mobile communications technology (2G, 3G etc), wire communications technology (Ethernet, BcN etc). For the final delivery of IoT services to the user, the developer also needs to apply service interface technology, which will cover wide range of functions: to sense, process/extract/treat, store, judge information; to recognize situation; to enhance security/privacy protection functionality; to certify and approve accesses; to conduct discovery, object standardization, open API and open platform etc.

The recent trends are pursuing inter-device communications in various wireless networks, whereby devices directly exchange information in infra-less environment. In cellular networks, the standardization has been undertaken to apply inter-device direct communication technologies from 3GPP to LTE (LongTerm Evolution) [7]. In unlicensed band areas, the Wi-Fi direct 802.1u technology has been developed along with the expansion of the existing wireless LAN standards [8],[9]. Further, in IEEE 802.15 .8 areas, the standardization for object recognition communications has been undertaken. The most significant advantages of inter-device direct communications are quoted as follows: first, it may substantially reduce lag times interfering in transmission, communication distance, and thanks to the bypassing of AP and base station engagement; second, it may create new environment for easy sharing of new types of proximity-based resources such as sensing information among IoT terminals, neighbor discovery, IPv6 addresses etc.

Nevertheless, there are certain additional requirements deriving from the specific characteristics of IoT environment-including low-power, light-weight and unstable communications setting etc. Most of all, the designs need to be undertaken in different perspectives apart from the existing ones in terms of automatic address setting, inter-device communications and routing protocols.

The proximity-based light weight automatic address setting and IPv6 neighbor discovery technique intends to achieve direct communications among IoT devices in circumstances where more than two IoT devices are physically located in proximate distance, which will become available through automatic address setting of light-weight IPv6 address to each IoT device, discovering neighboring devices and conduct direct communications among the devices bypassing the infra facilities. To realize these techniques, it is crucial to design the proper discovery protocols to identify the overlaps among the automatically-set IPv6 addresses on individual devices. Meanwhile, it is not easy by any means to design the protocols in ways enhancing the functions to discover the light-weight automatic address setting configuration and deliver the data to the destination within adequate time and with use of minimal wireless resources and least battery consumption.

In particular, in case multitudes of IoT devices fail to identify target IoT terminals and/or nonoverlapping IPv6 addresses, or fail to maintain the router path, then the devices should cut off the connection and repeat accessing again, which process will require transmission of large volume of unicast information-in short result in inefficiency.

Existing researches has focused on how to reduce overhead, as represented by CoAP (Constrained Application Protocol) [10] focusing on the solution in data transmission, RPL (Routing Protocol for LowPower and Lossy Networks) [11] designed to reduce control messages, Kunjan Patel, PSR, Hu She etc. The existing researches have concentrated on refined categorization of control message types, or setting of overall transmission path with the periodic exchange of beacons for a limited time span.

On the contrary, this paper proposes to achieve the reduction of overhead in the overall control messages by applying new schemes: first, to reduce the volume of messages among nods by utilizing hashfunction based bloom filter expression; and second, expressing the nod-recognizing addresses, neighbor discovery, routing path etc. in light-weight mode. When IoT terminals have light-weight addresses and involved in low-power lossy network on the basis of RPL system, the devices will be able to rerouting through flexible and small overhead path when a problem in looping and routing loss etc. occurs, which will enable the device to promptly identify the neighboring IoT terminal and resume solidly transmitting the data to the destination.

The proposed IoT terminal's light-weight automatic address setting, IPv6 neighbor discovery and light-weight routing protocol does not presuppose any specific communications technology and accordingly it may be subsequently applied to wifi direct, IEEE 802.15 .8 object recognition communication etc. This paper is composed as follows:

Light Weight Routing Protocol in IoT Based Inter Device Telecommunication Wireless.... (Hayoung Oh) 
In chapter 2, the relevant researches will be introduced and explained. In chapter 3, further explanations will be given to the enhanced RPL based light-weight routing protocol in the IoT-based interdevice wireless environment. In chapter 4, the results of performance assessment will be presented. Finally, in chapter 5, the conclusions and implications will be discussed.

\section{RELATED WORK}

The routing protocols for IoT-based low-power communications may be categorized based on control messages for path settings (control plane) and data transmission settings (data plane), which is largely presented in Table 1.

Table 1. IoT based protocols for Low-power communications

\begin{tabular}{|c|c|}
\hline Data Plan & Control Plane \\
\hline $\begin{array}{l}\text { 6LoWPAN (IPv6 over Low power WPAN) } \\
-\quad \text { Fragment Forwarding Technique } \\
\text { CoAP (Constrained Application Protocol) } \\
\text { - } \quad \text { M2M communication in constrained networks } \\
\text { - } \quad \text { Connect smart objects to the Internet } \\
\text { - } \quad \text { A bulk data transfer mechanism over UDP } \\
\text { - } \quad \text { Avoid fragmentation, retransmission of TCP to minimize state maintenance } \\
\text { and power usage } \\
\text { - } \quad \text { Its own loss detection and retransmission to avoid the problems TCP has in } \\
\text { - } \quad \text { Goal: HTTP equivalent for WSNs (Representational State Transfer: REST) }\end{array}$ & $\begin{array}{l}\text { RPL (Routing Protocol for Low power and Lossy } \\
\text { Networks) } \\
\text { - } \quad \text { Each node builds a directed acyclic graph } \\
\text { through which packets can be efficiently routed } \\
\text { to sink nodes } \\
\text { - From the sink, RPL builds routes to nodes } \\
\text { inside the network which can distribute data to } \\
\text { sensor nodes } \\
\text { - } \quad \text { contikiMAC used as radio cycling protocol } \\
\text { - energy consumption is measured using } \\
\text { Contiki's built in power profiler }\end{array}$ \\
\hline
\end{tabular}

\subsection{CoAP (Constrained Application Protocol)}

While the M2M related standardization is gaining momentum, the CoRE (Constrained RESTful Environments) working group in IEFT (Internet Engineering Task Force) has launched the standardization activities for CoAP (Constrained Environments Application Protocol), 6LoWPAN's high application protocol, since 2010. Figure 1 shows in what position the CoAP is located in the M2M standard architecture [12]. As shown in Figure 1, the physical class (PHY) and media-approach class (MAC) are based on the IEEE 802.15.4 standard for low-power sensor nod. 6LoWPAN protocol is located in Adaptation class for the interface with IEEE 802.15.4 standard and network class where IPv4 protocol belongs. CoAP is the application protocol for the higher transport among these standards and application class.

The nodes proper for CoAP application will have low-performance CPUs and low capacity RAM and ROM. It is a protocol for resource discovery, multicast support, asymmetrical tranjection request and response, which are based on Representational State Transfer (REST) for asymmetrical transmitting of an event corresponding to the conditions of devices constituting IoT devices.

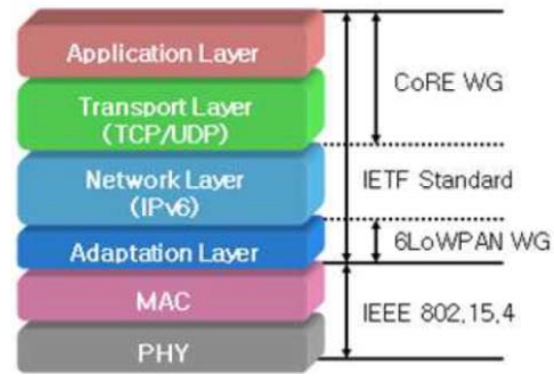

Figure 1. CoAP in M2M related architecture

The CoAP standardization areas purported by CoRE working group focus on how to design the methods of requesting resource events (e.g. temperature, humidity) to M2M nods in higher application class including the transport class with TCP and UDP; and in case of occurrence of an event, how to asymmetrically transmit the resource event to the nodes. While using HTTP, CoAP is different from the existing HTTP in that it supports both unicast and multicast in the UDP environment and sometimes its nodes may take the role of client, server and proxy. 


\subsection{RPL (Routing Protocol for Low-power and Lossy Networks)}

RPL (IPv6 Routing Protocol for Low-power Lossy Networks) refers to the IPv6 routing protocol that is being promoted for standardization by the IETF ROLL (Routing Over Low-power and Lossy networks) working group; hence, it is called as Ripple. RPL is designed to absorb all irregularities in network environment-such as low-power in IEEE 802.15.4 and electric wire communications, and noises etc.-and supports various routing matrixes to accommodate varying degree of requirements in numerous applications. For this purpose, a segment called Objective Function (OF) is created to cover routing matrix, path optimization etc. The definitions in the standard covers the general content commonly used in a number of OFs.

While the RPL objects have a destination-based direction, they are composed of several graphs (DODAGs: Destination Oriented Directed Acyclic Graphs) as shown in Figure 2. The ranks in nodes indicate the distance from the route DODGA. To compose DADAGs, each node periodically sends control messagesDAG information Object (DIO)-containing information on local links and ranks. The nodes receiving DIO will either participate in a new DODAG to select a parent nod for transmission at the least cost, or maintain the same DIO. Figure 4 shows the types of control messages in addition to DIO message, which are necessary for the operation of RPL.

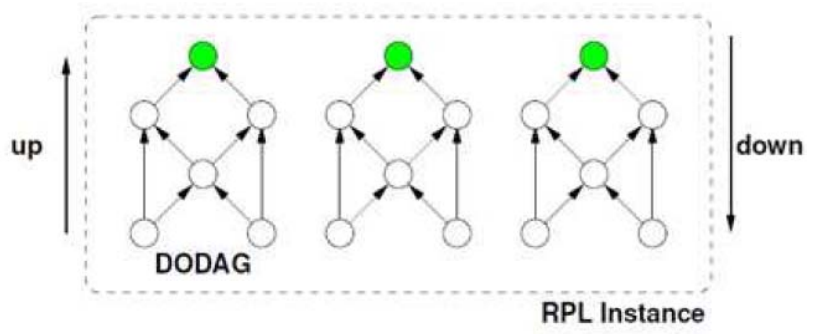

Figure 2. RPL object and destination based DODAGs: Destination Oriented Directed Acyclic Graphs

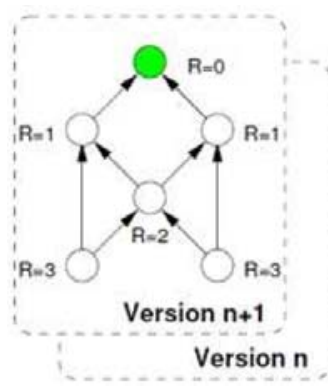

Figure 3. Rank and version definitions of DODAGs

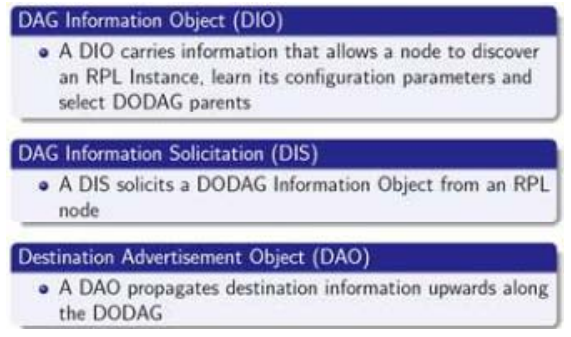

Figure 4. RPL control messages

RPL has three transmission techniques, i.e., data non-storing mode, data storing mode and a mode preventing data transmission to higher hierarchy. In the data non-storing mode, even in case of communications among the neighboring terminal nodes, the data should send upward to the DODAG root

Light Weight Routing Protocol in IoT Based Inter Device Telecommunication Wireless.... (Hayoung Oh) 
and then send downward to the receiving nodes. In data storing mode, the data may not to send upward to the root but bypass through neighboring higher nod and to the receiving nod. Regardless of the type, however, RPL is inherently exposed to the three problems as follows:

In the RPL method, nodes are put into sleeping mode for energy saving and thus they may fail to timely respond or are susceptible to low-power and data loss, which may all together lead to a low rate of data success. To cope with this problem, the RPL method utilizes DIO control messages6 in case of occurrence of any problem in data transmission.

The RPL method may not completely solve loop problems. Accordingly, whenever a loop intervenes, it is necessary to solve it in quickest manner through loose avoidance with real-time detection, utilization of DIO, DIS and DAO control messages calling for small overheads. For instance, RPL intends to transmit data only to a path with decreasing ranks, whereby loop may be avoided. On the contrary, if data is transmitted to a path with increasing ranks, the probability of loop occurrence will be heightened. It is crucial, therefore, that the Objective Function should be properly designed to accommodate routing matrixes and path optimization to meet the requirements of each application, for which the designer should determine whether the problem will be surgically solved within the confine of the area or to be solved by referring to the overall system.

RPL has proposed a technique to transmit data at low power in dynamic environment as opposed to ISA 100.11a or wireless HART. Still, however, RPL is deemed to be ill-equipped to handle the problem of data disconnection. It attempts to solve it through DIO positioning, which is deemed to be imperfect as it incurs overheads for maintaining and updating DODAG each time a problem occurs.

The LLN (Low-power and Lossy Networks) router refers to low-power loss networks. This scheme is subject to the constraint deriving from the use of limited memory, which makes it hard to maintain the destination path information in excess of the small amount of default router information. For instance, under the IPv6 based network protocol standards in IEEE 802.15.4, the environment comprises low-power, lowprice, low-capability (8 bit-microprocessors, one digit KB RAM) and low speed (up to 250 kbps) and accordingly is constrained to limited scope of devices as presented in Table 2 for sizes and Table 3 for roles.

Table 2. Device classifications based on the limited memory size

\begin{tabular}{ccc}
\hline & Data Size & Code Size \\
\hline Class 1 & $\sim 10 \mathrm{~KB}$ & $\sim 100 \mathrm{~KB}$ \\
Class 2 & $\sim 50 \mathrm{~KB}$ & $\sim 250 \mathrm{~KB}$ \\
\hline
\end{tabular}

Table 3. Device role based classification with the limited memory

\begin{tabular}{|c|c|c|}
\hline Constrained nodes & Nodes talking to constrained nodes & Gateways/Proxies \\
\hline - $\quad$ Sleepy nodes & $\begin{array}{ll}\text { - } & \text { To sleepy nodes } \\
\text { - } & \text { Normally always alive }\end{array}$ & $\begin{array}{ll}\text { - } & \text { To sleepy nodes } \\
\text { - } & \text { Could be always alive }\end{array}$ \\
\hline
\end{tabular}

In consequence, it is required that the LLN router in constrained environment should be able to maintain the connectivity information and the path information for the transmission of datagram. It also needs IPv6 source routing for the transmission of diagram. Mindful of this, this paper will focus on the development of the technique to find IPv6 neighbors (including IoT terminal's light-weight automatic address setting) and light-weight routing protocols, which are essential for inter-RPL routers transmission of datagram in Infra-free environment.

\subsection{Other Light-Weight Routing Protocol}

Kunjan Patel has proposed a light-weight routing protocol with the effect of reducing unnecessary messages by making the routing protocol be activated only with various replying messages from unicast operation in a sensor network (acknowledgement) and within time limits (timeout schemes). This approach has still a lot of control messages to be exchanged in total since data may be exchanged within limited time span and the performance improvement is constrained.

[13] has proposed a light-weight source routing protocol (PSR: A Lightweight Proactive Source Routing Protocol For Mobile Ad Hoc Networks) that is capable to know all paths from the source to the destination in mobile wireless network environment. This technique operates on a binary-tree based routing protocol enabling to utilize larger information on topology through small overheads. This technique is, however, limited since it is operable only on a binary-tree based routing protocol. Due to the total volume of control messages to convert topology into a binary-tree system, the overall amount of control messages is still substantially large.

IJECE Vol. 6, No. 5, October 2016 : 2352 - 2361 
Hu She [14] proposed a technique of collecting real-time local traffic information in a light-weight mode, which is applicable in an automobile wireless network environment. For this objective, Hu She suggested to apply a technique effectively monitoring the link conditions and transmitting data through periodic beacon messages. In wireless network environment surrounding a moving automobile, it is available to transmit data to the destination with the minimum number of transmission; nevertheless, to perform the intended function, the system should periodically exchange control messages, confirm the link status and store the information, whereby the total volume of control messages to be transmitted and stored may not be significantly reduced. The existing researches have focused on the categorization of control message types for light-weight transmission of data to the destination, or resetting the overall path for data transmission through periodic exchange of data.

On the contrary, this paper proposes to utilize bloom filtering expression to the effect of reducing the volume of data exchanges and further achieving light-weight in node recognition address, neighbor discovery and routing path, which has all together resulted in a love level of overhead of total control messages. In particular, the proposed method has-for the first time-composed the channel conditions incurring a lot of data loss and conducted the performance testing; this scheme enables the devices to counter an occurrence of data loss by rerouting to multiple paths available in the local area by referring to the enhanced ranking techniques. It was demonstrated that this scheme has achieved up to $40 \%$ performance improvement in comparison to the existing RPL routing protocols.

\section{ENHANCED RPL-BASED LIGHT WEIGHT ROUTING PROTOCOL IN IOT-BASED INTER- DEVICE WIRELESS COMMUNICATIONS ENVIRONMENT}

\subsection{Light weight IPv6 address setting on the basis of bloom filter and enhanced ranking method}

The rapid increase in IoT devices and support for hyper-connectivity will require a larger volume of message exchanges related to sensing data, IPv6 address and neighbor discovery. In the meantime, IoT terminals and LLN routers are related to ultra-lightness, low-power and loss occurrence; hence, such devices should store a minimum amount of data. Further, a solution needs to be found to meet the requirement to transmit data through a consistent path to the destination. It is necessary, therefore, that addresses should be compressed to the maximum by utilizing a light-weight notation method.

For the setting of light-weight IPv6 addresses, this paper utilizes the bloom filter and an enhanced ranking method. In the existing RPL methods, in order to identify the level of data transmission, rank has been utilized as a scale indicating the depth from the root node. Contrary to this, this paper adopts a new concept of rank ('enhanced rank'), which includes additional information referring to a relative location. For this purpose, a new second index is applied to indicate the location of a child node apart from the parent nod. For instance, in $(3,0)$ the rank is 3 , indicating its location to the left from the relative parent node $(2,0)$. In $(3,1)$, the rank is also 3 , yet it is located to the right side of the $(2,0)$ node.

In the RPL method, the neighboring nodes with the same rank means that they are placed on the same level from the root node. Accordingly, in order to avoid loops and solidly transmit data, the path should be concentrated on the neighboring nodes on lower ranks. The proposed enhanced RPL (eRPL) method will ensure that data shall be transmitted through a solid path along incessant combinations regardless of the value of relative locations, provided the ranks are same or lower. As indicated in Figure 5, the proposed method utilizes light-weighted IPv6 addresses by referring to the 3 multiple paths from the source node to the destination.

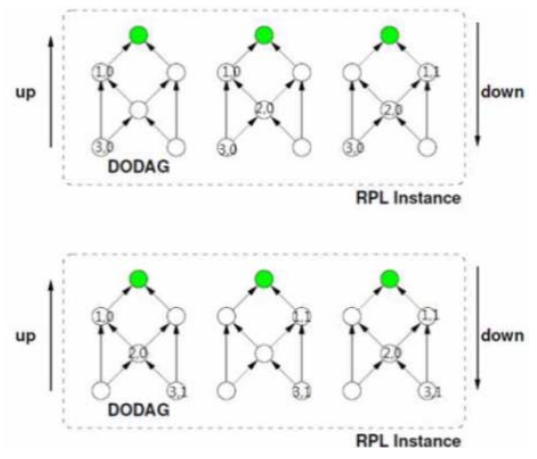

Figure 5. An enhanced RPL based light-weight routing protocol 
Figure 6(a) shows the process of identifying nodes on the basis of a node identifier created through the bloom filtering, which will replace IPv6 addresses. For instance, Node 1 indicates location 1, 6 and 10 set at 1 bit; Node 2 location 2, 3, 7 set at 1 bit; Node 3 location 2, 3, 8 set at bit 1 . If a path-setting controlmessage packet is transmitted from the source to the destination through Node 1, 2 and 3, the bloom filter at the destination will have the final results location $1,2,3,6,7,8,10$ all set at 1 bit. The destination will compare the bloom filter of neighboring nodes with the final bloom filer containing locations as checked 1 bit.

Figure 6(b) shows a multi-hub scenario wherein each node is expressed in the 10-bit bloom filter in a condition that there are multiple paths transmitting data from the source to the destination. Figure 6(c) shows the procedures how the bloom-filter table manages each of the multiple paths transmitting data from the source to the node $(1,0)$ immediately prior to the destination node.

However, there are limitations with the bloom filter values in that the values may overlap or be inaccurate in the degree of recognition. Accordingly, the proposed method solves this problem by additionally utilizing the enhanced rank that is to identify the relative location of child nodes. In the relevant codes, the information contains compressed notations naming the neighboring nodes, which make it impossible to recover the full names of neighboring nodes. Instead, the compressed notation allows the destination to confirm whether one specific neighboring node's name is included in the set of the neighboring nodes list of the sender. This means, of course, that an error may be invited due to the loss of information while testing the existence of the neighboring nodes by referring to the bloom filter code.

If it is judged that the neighboring nodes exist while the truth is 'not', it is an error false negative. To prevent a failure in discovering the neighboring nodes, the bloom filter codes have been designed in a condensed format reflecting the enhanced ranks. In other words, even in case there are several neighboring nodes belonging to the same bloom filter code, they are different in the child node locations relative to the location of the parent nodes; accordingly, the enhanced rank identifier will be able to solve the inaccuracy of the bloom filter codes.

In consequence, the proposed method will be able to discover the light-weighted IPv6 addresses and neighboring nodes and set light-weighted routing protocols by utilizing bloom filer codes containing information relating to the overall path and relative locations of child nodes. Unlike the existing RPL methods, the proposed method maintains various child paths whose parent nodes are implied in bloom filter codes, whereby it will counter errors in IoT-based wireless networks by referring to other available lightweighted paths in the local area.

\subsection{Light-weighted Neighbor Searching Method on the basis of bloom filter}

In general, a communication terminal needs to send periodic signals-e.g. beacon-containing its identifier to identify any neighboring terminals and send messages containing information about the neighboring nodes so that they may be discovered. Nevertheless, signals containing the name and list of neighboring nodes will necessitate heavy amount of information, which is not suitable for a low-power lossy network environment.

In the proposed method, to reduce the number of bits required to announce the neighboring node list, the information relating to the neighboring nodes on the path linking the source and the destination will be expressed in condensed bloom filer codes and managed in the bloom filter table. For instance, the bloomfiler based neighbor management table contains the child nodes-(2,1), $(2,0),(1,1)$-and the destination node. In case of Node (1.0), the Node's bloom filter values indicating the relative location of each child node and the bloom filter value implying the overall path including the intermediary nodes through transmission process, will be managed by the relevant table. If Node $(1,0)$ has received a bloom filter data, as expressed 1111000000 , the bloom filer values of the neighboring node from Node $(1,0)$ may include only one, i.e. (0111000000) from the candidates of (00000110101), (0111000000) and (0000011001). Therefore, it will be identified that the data has come from the source, to $(3,1)$, to $(1,1)$ and finally to $(1,0)$. 


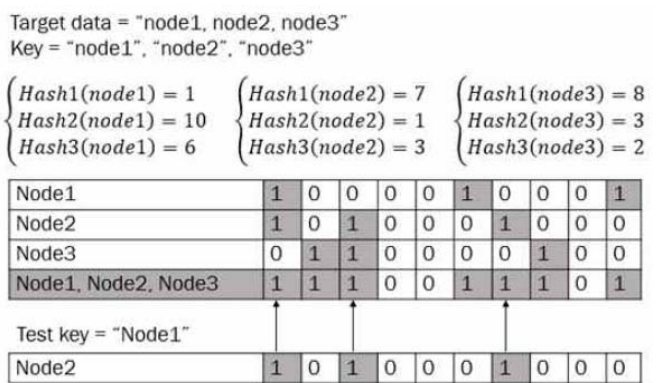

(a)

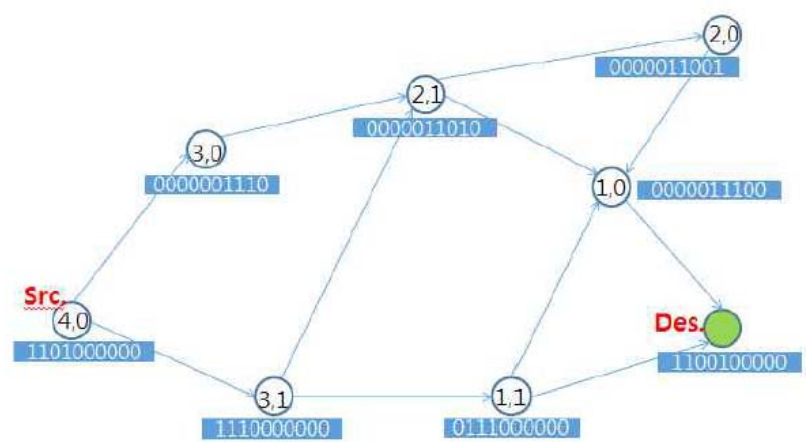

(b)

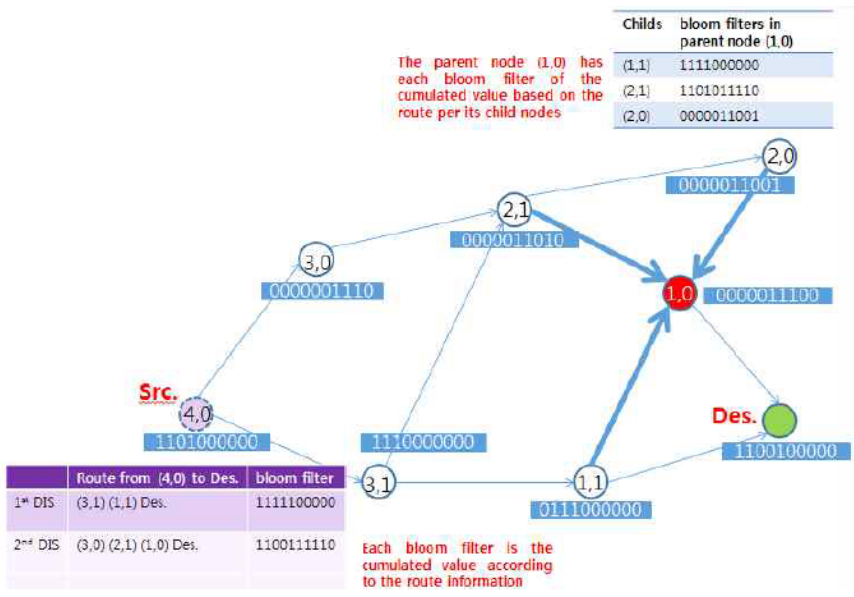

Figure 6. (a) Bloom filter based light-weight IPv6 address, (b) The multi-hop scenario with Bloom filter based light-weight IPv6 addresses (c) Light-weighted various routing routes establishment in eRPL

\section{PERFORMANCE EVALUATION}

For the performance assessment of the proposed method, NS-3 (Network Simulator-3), one of event-driven simulators, has been utilized to assess the results from the setting of low-power lossy multi-hub network in the no-storage mode, as presented in Figure 6(c). To create a lossy network environment, various scenarios have been considered to reflect the probability of link transmission failure-normal, log-normal or mixed distribution; in case of non-existence of data transmission path, the path has been rerouted to the DODAG root by utilizing the local scheme (local repair scheme), the overall scheme (best average repair scheme) or the simultaneous application of both schemes (local and best average repair scheme).

The local repair scheme is a kind of greedy method, focusing on the best link among the neighboring node wireless links; the best average repair scheme intends to select the overall favorable wireless link from the source to the destination; the local and best average repair scheme utilizes the mixed pool of both techniques.

The RPL method belongs to the best average repair scheme wherein DODAG is produced in various versions and ranks are set thereto. If we assume occurrence of problems such as nodes in random sleeping

Light Weight Routing Protocol in IoT Based Inter Device Telecommunication Wireless.... (Hayoung Oh) 
mode, link channel errors, loop problems, we are deemed to face limitations in solving the problems through dynamic methods. In contrast, the proposed method (local \& best average repair scheme) has added a new local solution (local repair scheme) capable to utilize various paths on the basis of enhanced ranks considering the relative locations. The results are at least 40 percent improvement in performance.

Figure 7 shows the cumulative distribution function in case the wireless link errors are related to the normal distribution; Figure 8 shows the cumulative distribution function in case the same errors are related to the log-normal distribution.

The performance of three methods has been assessed against the optimal repair scheme in the situation where the wireless link errors display dynamic movements on the basis of each distribution function. It is intuitively apparent that the proposed method (local \& best average repair scheme) shows the least differences from the optimal repair scheme since it utilizes the opportunities in transmitting data by referring to other path from the multiple options in the local area in case of occurrence of wireless link loss in the original path.

Given the IoT based wireless inter-devices communications environment is not fee from frequent error occurrence, the method considering local values rather than average values will have higher level of applicability and good performance.

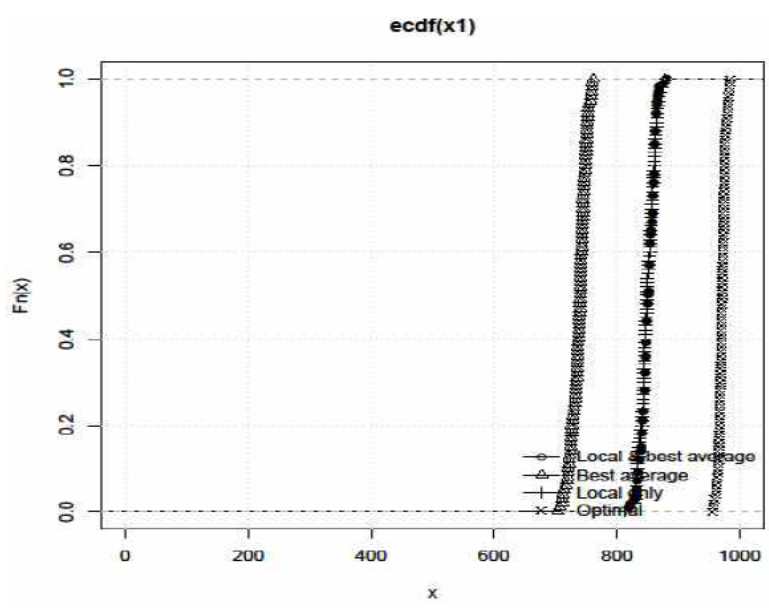

Figure 7. CDF with the normal distribution of wireless link errors

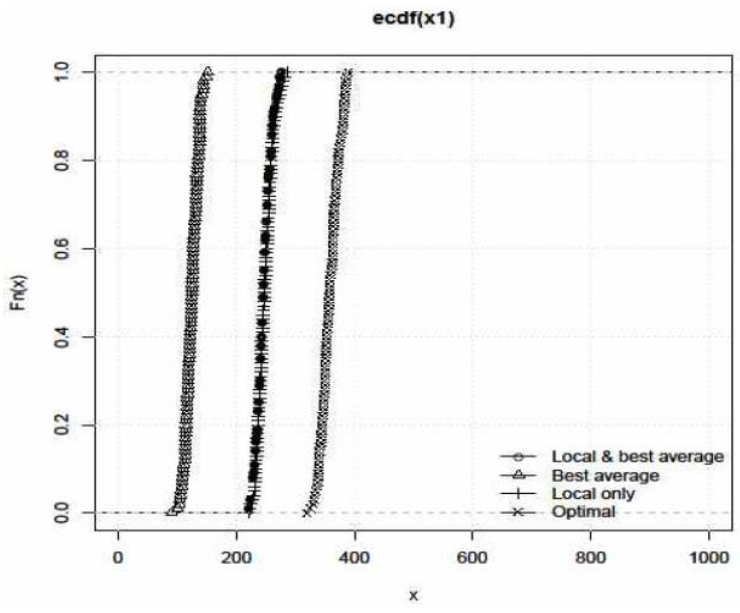

Figure 8. CDF with the log-normal distribution of wireless link errors

\section{CONCLUSION}

This paper has proposed IPv6 neighbor discovery method including IoT devices’ automatic lightweight address setting and enhanced RPL-based light weight routing protocol in the IoT-based wireless inter- 
device communications environment. The existing researches have proposed light weight routing protocols resorting to the reduced frequency in control message transmission and the size of transmission packet. However, this paper proposed a RPL-based enhanced routing protocol that will effectively reduce the total amount of control messages deriving from node-identifying IPv6 address, neighbor discovering procedures, and routing path in the data transmission.

\section{ACKNOWLEDGEMENTS}

This research was supported by Basic Science Research Program through the National Research Foundation of Korea (NRF) funded by the Ministry of Science, ICT \& Future Planning (NRF2014R1A1A1003562).

\section{REFERENCES}

[1] Everything, "White Paper: Everything connected," 2012.

[2] G. Chrisman, "IBM Innovations and Smarter Planet projects,” 2012.

[3] G. Fodor, et al., "Design aspects of network assisted device-to-device communications," IEEE Commun. Mag., vol/issue: 50(3), pp. 170-177, 2012.

[4] CISCO, "Smart+Connected Communities-Changing a City, a Country, the World," 2013.

[5] Ji Z., "Design of an integrated controller based on ZigBee wireless sensor network," TELKOMNIKA Indonesian Journal of Electrical Engineering, vol/issue: 11(8), pp. 4414-4421, 2013.

[6] N. Dinh and S. Lim, “Analysis of IEEE 802.15.4 Beacon-Enabled MAC Protocol,” IJECE, vol/issue: 6(3), 2016.

[7] L. Lei, et al., "Operator controlled device-to-device communications in LTE-Advanced networks," IEEE Wireless Commun. Mag., vol/issue: 19(3), pp. 96-104, 2012.

[8] D. C. Mur, et al., "Device-to-device communications with Wi-Fi Direct: overview and experimentation," IEEE Wireless Commun., vol/issue: 20(3), pp. 1-8, 2013.

[9] IEEE, "Wireless LAN medium access control (MAC) and physical layer (PHY) specifications amendment 9: interworking with external networks,” IEEE Std. 802.11u, 2011.

[10] Z. Shelbyet, et al., “Constrained Application Portocol (CoAP),” IETF CoRE Working Group, Internet-Draft, ver. 18, June 2013.

[11] IETF, “RPL: IPv6 Routing Protocol for Low-Power and Lossy Networks,” RFC 6550, Mar. 2012.

[12] IETF, “Transmission of IPv6 Packets over IEEE 802.15.4 Networks,” RFC 4944, Sep. 2007.

[13] Z. Wang, et al., "PSR: A Lightweight Proactive Source Routing Protocol For Mobile Ad Hoc Networks,” Vehicular Technology, IEEE Transactions on, vol/issue: 63(2).

[14] H. Shen, et al., "LALO: A Link-Aware Lightweight Routing Protocol for Data Delivery in Vehicular Ad Hoc Networks,” Ubiquitous Intelligence and Computing Lecture Notes in Computer Science, vol. 6406, pp. 459-473, 2010.

\section{BIOGRAPHIES OF AUTHORS}

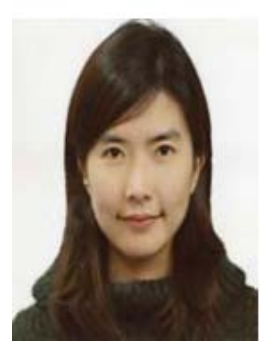

Hayoung Oh received the B.S. degree in Computer Science from Duksung Womans University and the M.S. degree in the School of Computer Science and Engineering from Ewha Womans University in 2002 and 2006 respectively. And she received the Ph.D. degree in Computer Science from Seoul National University in 2013. From 2002 to 2004, she joined Shinhan Financial Group as a developer in applied research. In 2010, she was with U.C. Berkeley as a researcher. Since 2013, she has been with Soongsil University as a professor in the School of Electronic Engineering. Her research interests include social and computer networks, and security.

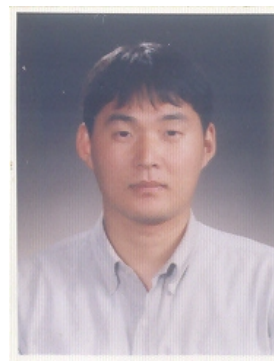

Sangsoon Lim received Ph. D. degree in the School of Computer Science and Engineering from Seoul National University in 2013. Since October 2013, he works as a senior engineer at Software R\&D Center, Samsung Electronics. His current research interests are in the area of wireless networks including wireless LAN, wireless sensor Networks, cognitive radio networks. 